\title{
Humanización EN LA GeSTión de PROYECTOS INFORMÁTICOS EN LAS UNIVERSIDADES
}

\author{
HUMANIZATION IN THE MANAGEMENT OF COMPUTER PROJECTS IN UNIVERSITIES
}

Ph.D. Fidel Parra

Universidad Tecnológica Israel

fparra@uisrael.edu.ec

Lcda. Mayoribel Reañez

Universidad Tecnológica Israel (estudiante de la Maestría en Telemática)

maryoribel20@gmail.com

Dra. Mercedes Delgado

Universidad de Zulia

merdelgon@hotmail.com

\section{Resumen}

Fecha de recepción: 04/08/2016

Fecha de aceptación: 07/10/2016

El propósito de esta investigación fue analizar la humanización en la gestión de proyectos informáticos en las universidades de Ecuador y Venezuela. La investigación está sustentada en autores como Alcalá y otro (2013), Hewitt (2008), entre otros; se enmarca en el paradigma cualitativo, siendo en tal sentido el tipo de estudio documental, analítico, realizado a través de la técnica de la observación directa sobre la lectura referencial recopilada y el análisis de contenido para la interpretación de los referentes conceptuales desarrollados. 
De dicha revisión documental se localizaron debilidades referentes a la ausencia de innovación, de modernidad, de asimilación tecnológica, de aprendizaje permanente, de acciones efectivas para el aprovechamiento de equipos y recursos hardware y software en instituciones universitarias. En razón de ello se sugirió la sensibilización de los actores educativos en el sentido de promover las buenas prácticas en los entornos tecnológicos existentes en esas casas de estudios, con lo cual sería posible la sistematización de los equipos informáticos, su buen uso, para la generación de proyectos y la solución a inconvenientes administrativos y académicos en esas casas de estudios.

Palabras clave: Humanización - Gestión - Proyectos informáticos - Universidades - Sensibilización - Buenas prácticas.

\begin{abstract}
The purpose of this research was to analyze the humanization in the management of computer projects in universities. The research is supported by authors such as Alcalá and other (2013), Hewitt (2008), among others; it is part of the qualitative paradigm, being in this sense the type of documentary, analytical study, carried out through the technique of direct observation on the reference reading collected and the content analysis for the interpretation of the conceptual references developed.

From this documentary review, weaknesses were located related to the absence of innovation, modernity, technological assimilation, lifelong learning, effective actions for the use of equipment and hardware and software resources in university institutions. As a result of this, it was suggested to raise the awareness of the educational actors in the sense of promoting good practices in the technological environments, existing in these houses of study, which would make possible the systematization of computer equipment, its good use, for the generation and the solution to administrative and academic disadvantages in those houses of studies.
\end{abstract}

Keywords: Humanization - Management - Computer projects - Universities - Sensitization - Good practices. 


\section{Consideraciones previas}

El cambio de actitud y la mejora de las aptitudes del talento humano se ha constituido en un tema de relevancia en las organizaciones modernas, si se parte de la nueva concepción de la humanización de los procesos, buscando evitar la rutinización, el retardo en las tareas, la ineficacia, la apatía, la resistencia a las constantes transformaciones suscitadas en esta era. Es así como, con la evolución de las figuras gerenciales en las organizaciones, se ha implementado la idea de conducir las actuaciones de sus miembros a través del aprendizaje de la vida humana, para inducir el cumplimiento de objetivos comunes.

Más aún, en tiempos de globalización, que Morin (1998) llama mundialización, se determinan exigencias que las empresas deben cumplir, en virtud de la necesaria productividad y competitividad; no obstante se observa que en algunos casos, en la actualidad, no se haya advertido el capital humano como canalizador del éxito.

En tal sentido, se requiere la generación de valor agregado, la capacidad de internacionalización, la asociatividad, amparados bajo la inclusión de la ciencia y tecnología, convertidos en factores claves de competitividad que determinan la permanencia de las empresas en un entorno cada vez más globalizado, en el cual juegan un papel protagónico las diferentes formas que toman las relaciones sociales de producción, y dentro de ellas, el manejo, administración y gestión del talento humano en lo tecnológico, que es, en definitiva, el eje central de cualquier organización.

Es así como en el escenario mundial, según plantea Hewitt (2008) la globalización ha conllevado a la necesidad de la gerencia de interpretar su rol sobre el éxito en el ejercicio de sus funciones, pero tratando de gestar, a la par, un nuevo paradigma de humanización de la empresa, sobre la base del establecimiento de la eficacia tecnológica, para conllevar transformaciones sociales consistentes.

A los mismos efectos, en el ámbito latinoamericano, consideran Calderón, Cuartas y Álvarez (2009), que existe un factor de éxito de las organizaciones, que debe adecuarse al contexto global que se vive actualmente en el mundo, y es la capacidad para cambiar de manera apropiada, trascendiendo la idea de adaptación para llegar al de transformación tecnológica. 
Esto es, desarrollar la capacidad para modificar no sólo estructuras, sino también para replantearse elementos más profundos, como valores, comportamiento, cultura y métodos de operación, manteniendo una actitud de renovación y aprendizaje permanente. Para lograrlo se requiere comprender las transformaciones organizacionales como procesos humanos, antes que técnicos o ambientales; en este sentido, la gestión humana tiene un papel fundamental en su éxito.

Es así como instituciones de educación superior, como es el caso de la Universidad de Pamplona (2016) en Colombia, fomenta el desarrollo de la gestión de proyectos, la ingeniería del software y la planeación estratégica de sistemas de información, que repercutan en soluciones viables a problemas regionales, nacionales e internacionales, pero mediante la utilización de las buenas prácticas. Incluso, se pretende la formación de investigadores integrales de profundización en el campo de la gestión de proyectos informáticos, que inciden positivamente en el desarrollo social y el mejoramiento de los procesos a las soluciones informáticas de gestión en diversos contextos, pero que hagan uso de experiencias humanas axiológicas, valorativas, lo que induce la gestión humana en los perfiles de egreso.

No obstante lo planteado, se observa en la literatura revisada que en la praxis directiva de algunas instituciones de educación superior, se suceden esfuerzos aislados, los cuales resultan insuficientes para generar transformaciones en razón de haber realizado la adquisición de equipos tecnológicos, pero no su asimilación efectiva.

Igual situación se evidencia tanto en el caso de Ecuador como en Venezuela, que las organizaciones modernas han debido suscitar una serie de transformaciones ante la vorágine de los tiempos, los innumerables cambios políticos, jurídicos, sociales, económicos, culturales, suscitados en su entorno, para buscar la humanización de la gestión tecnológica, su reinvención, además de factores innovadores en su hacer y un proceso de aprendizaje permanente, además del desarrollo de competencias personales y profesionales de los sujetos implicados.

Por eso, para dar cumplimiento a sistemas y estructuras organizacionales de esa magnitud, deberán los actores plegarse a una gestión basada en la humanización, de acuerdo al criterio de Morán (2017), en la ética y encuadrada en un enfoque estratégico, con cuyas perspectivas sea posible sensibilizarse en el fomento de las buenas prácticas comportamentales, morales, excelentes condiciones de trabajo, y el cuidado y mantenimiento de sus zonas de desenvolvimiento.

Lo planteado en el criterio del autor mencionado conllevará su propia satisfacción laboral, mejorará los escenarios de atención al público, logrando que se cumpla con la misión de servicio y la visión de posicionamiento estratégico de cualquier entidad, incluyendo las instituciones universitarias, a quienes se les plantea el desafío de adecuar las bondades tecnológicas a su desempeño, en ambientes agradables, amistosos, donde los procesos se simplifiquen, se resuelvan las dificultades y se dé cumplimiento a las metas de calidad y pertinencia universitarias.

Ese estilo de trabajo, debe derivar de la percepción de la realidad del sujeto interviniente en la empresa, a la vez de su responsabilidad, formación para el cambio, del desarrollo de su resiliencia o capacidad para convertir 
la adversidad en oportunidades personales o empresariales y para el afrontamiento de retos o desafíos que los mismos cambios de los tiempos suscita. Esto implica su propia sensibilización al cambio, la humanización de sus procesos en la tarea a cumplir, el ejercicio de su gestión con transparencia y la eficiencia en los resultados.

No obstante los precedentes evidenciados, es el caso que se ha venido observando en algunas dependencias universitarias tanto ecuatorianas como venezolanas, específicamente en las ciudades de Quito y Maracaibo respectivamente, que no siempre se produce la sinergia necesaria entre los actores educativos para efectuar los procesos de planificación, seguimiento y control de los proyectos software. De la misma manera, los investigadores observan que se trabaja de manera aislada, no implementando metodologías cooperativas, ni tecnologías donde se produzca la integración de los sujetos intervinientes.

Por otro lado, se observa el uso indiscriminado de los equipos tecnológicos, sin mayor cuidado, sin darles el mantenimiento adecuado. Produciéndose una gestión del talento humano separada de una cultura constructiva, desde donde se puedan ejecutar los actos, las tareas necesarias, a través de la concepción de las buenas prácticas y la preservación de los insumos tecnológicos existentes en las universidades examinadas, para su aprovechamiento en términos de tiempo y espacio.

En igual sentido de lo expresado, se observa la problemática acerca del retardo en el cumplimiento de los requerimientos de usuarios de los sistemas tecnológicos, no se atienden las exigencias a tiempo, desequilibrando el tiempo de la entrega de los trabajos administrativos o académicos. Asimismo, se evaden responsabilidades cuando los equipos se dañan o cuando no son lo necesariamente operativos.

De la misma manera, en las universidades no siempre se producen acuerdos entre las personas involucradas en las responsabilidades tecnológicas, para usar un mismo lenguaje o para utilizar procedimientos y documentos estandarizados, en el afán de lograr resultados eficaces para la organización. No produciéndose de igual modo, hasta la fecha, la concienciación sobre la importancia de la profesión informática, del diseño y utilización de patrones o estándares de desempeño convenientes, adecuados a la organización universitaria, además de no atender a los aspectos legales y éticos de las tecnologías de la información y la comunicación, para asumir la responsabilidad social y jurídica que les compete.

De allí que se pretenda en este estudio analizar la humanización en la gestión de proyectos informáticos en las universidades, buscando examinar hasta qué punto se han venido produciendo tales acciones tecnológicas en instituciones de estudios del nivel superior, sobre la base de una gestión humana que apuntale principios axiológicos en su desempeño.

\section{Gestión humana en la organización}

En la actualidad, la gestión humana es un componente esencial de toda organización y más que un conjunto de acciones, obedece a un proceso integrador, desde cuya perspectiva debe darse la administración estratégica del 
recurso humano, como la denomina Dolan (2013), teniendo como finalidad dotar a los directores de recursos humanos y a todo el personal de las competencias necesarias para integrar esa gestión humana a la gestión general de la organización.

En este sentido, será necesario, en el caso de las universidades examinadas en este estudio, identificar, formular y aplicar políticas de recursos humanos para toda la organización, con la finalidad de sensibilizar al personal en buenas prácticas y mejores formas de desempeño; pero más allá de ello, debe concebirse la organización universitaria desde una perspectiva sistémica y multidimensional, en la que se integren diferentes tendencias, estrategias y técnicas de gestión humano-tecnológica, que hagan posible una administración en la que se ya no se aliene, sino que se rehabilite el sujeto - actor en la transformación organizacional.

Consideran los investigadores en este estudio, que las universidades requieren en la actualidad canalizar sus sistemas informáticos, su gestión de proyectos tecnológicos con la finalidad de modernizar su base de datos, sus estructuras internas, incluyendo la modernización de los equipos para impedir la obsolescencia de los mismos, a partir de las directrices y orientaciones de una gestión humanizada, centrada en el poder del individuo en la empresa, en el empoderamiento de sus tareas desde la visión de los principios organizacionales, y en general de una filosofía corporativa guiada por valores estratégicos de unidad, orden, disciplina y responsabilidad.

\section{Humanización en los procesos organizacionales tecnológicos.}

En las organizaciones se debe atender a una visión estratégica de servicio, si lo que se pretende es la puesta en práctica de una gestión humana, buscando en definitiva, de acuerdo al criterio de Pernía (2017), articular todos los elementos relacionados con categorías centrales como la prospectiva estratégica, en el ánimo de determinar la visión de futuro, y la gestión enfocada en el capital humano.

Considera el mencionado teórico que tales elementos deben estar referidos a la generación del cambio en todo momento, como un eje perpendicular en todos los procesos efectuados. Asimismo, se deben concretar los business-plan de las organizaciones de manera novedosa, comprometidos con las organizaciones examinadas, canalizados sobre la base de una dirección estratégica efectiva, que conozca del negocio, que sepa encaminar los resultados deseados, poniendo en práctica las estrategias motivacionales necesarias al éxito y la consecución de las metas planeadas.

Desde esa óptica se hace énfasis en que lo planteado debe ser apuntalado por la humanización de los procesos, sobre todo informáticos, en los cuales reposa la sistematización de la información empresarial proclive a la toma de decisiones de la misma; debiendo entender, comprender las acciones de los colaboradores desde la perspectiva de la integración de criterios, produciendo sinergia para sumar voluntades a la necesaria competitividad empresarial, que lleve a las organizaciones a un posicionamiento, hoy local, mañana regional, nacional y mundial, que ha de ser construido de esa manera global. 
Por su parte, Escorsa, Maspons y Ortiz (2000) opinan que la gestión de conocimiento de la organización tiene una herramienta estratégica denominada mapas tecnológicos, que permitirán hacer un diagnóstico de la situación actual de la organización y del entorno, estableciendo cuáles serán los elementos que pudieran incorporarse para mejorar la productividad de los trabajadores y los factores o los procesos de cada una de las cadenas de valor de las empresas.

Asimismo, la tecnología permite organizar la información a manera de poder visualizar los elementos necesarios para una toma de decisiones efectiva que conlleve al logro de los objetivos de la empresa y sirva para consolidar la plataforma tecnológica de la empresa en términos de la gestión de los talentos, los recursos y procesos.

De este modo, el manejo de la tecnología dentro de la organización representa una ventaja competitiva para el talento humano formado para ello, lo cual genera a la empresa beneficios en su actuación, debido al aumento de su rentabilidad y su productividad, devenido del desempeño efectivo, y del rendimiento producido por su capital intelectual, lo cual constituye su acervo más importante. La gestión del conocimiento en la que se busca desarrollar las competencias del talento humano, contribuye a apropiarse y a consolidar el proceso de decisión gerencial, la cual se apoya en la vigilancia tecnológica y a través de mapas tecnológicos.

\section{Humanización y ergonomía}

Las últimas tendencias organizacionales consideran como objetivo primordial de las empresas e instituciones del mundo, alcanzar una mayor humanización del trabajo a través del diseño de puestos de trabajo más ergonómicos, generando organizaciones eficaces, más democráticas y participativas capaces de satisfacer las necesidades, así como las demandas de sus miembros, además de ofrecerles oportunidades de desarrollo profesional y personal.

Así lo señalan Segurado y Agulló (2012), quienes aseveran que en el entorno mundial de negocios, se desarrolla una competencia feroz, con múltiples alternativas de productos y servicios los cuales deben satisfacer las necesidades reales que el mismo mercado genera; pero, que las empresas deben enfrentar nuevos retos de calidad tanto en procesos como en sus ofertas al cliente, atender a los desafíos de eficiencia en la gestión y reducción de costos de la innovación tecnológica generando la productividad en general.

De esta forma, dado que la competitividad, las condiciones, ambiente de trabajo y la calidad de vida del trabajador marchan paralelamente, es difícil mantener una empresa en el mercado si los procesos de trabajo deterioran la salud de los trabajadores generando conflictos. De allí que deben surgir mecanismos humanizadores en las organizaciones, buscando traducir resultados en bienestar psicológico y calidad de vida.

Estos aspectos tienen repercusiones significativas en el equilibrio socio-emocional así como el afectivo de los miembros de la empresa, es decir, en su bienestar psicológico, e impactan en la productividad y la 
perpetuación de la compañía. De allí que resulta de importancia asumir el compromiso, la confianza, la responsabilidad, la ética, como factores clave de la gestión humana, como aspectos propicios al desarrollo de la calidad de vida laboral.

Este último referente ético se muestra, en las entidades y organizaciones modernas, según Nuváez (2016) como una estrategia imprescindible en el logro de la humanización de las sociedades actuales, en las cuales deben privar los valores humanos como piezas clave para el logro de una paz duradera en aquellos pueblos donde se pretenda el respeto por la vida y los bienes personales de subsistencia.

En igual sentido de lo expresado, Alcalá y Conti (2013) aseveran que la productividad de las organizaciones ha devenido en un tema de extendida discusión a nivel gerencial, siendo muchos los elementos a los cuales se les atañe el aumento o disminución de este índice tan importante. Pero, en los últimos años se introdujo la medición de la productividad en la calidad del espacio laboral, razón por la cual ciencias incipientes como la ergonomía, el diseño de ambientes laborales dignos, incluso la tecnología para humanizar aún más las tareas y responsabilidades se han puesto en el tapete de los estudiosos de las ciencias gerenciales.

De allí que ha de atenderse a ciertos indicadores de humanización del trabajo, al marketing interno y la tecnología como base de la productividad social empresarial, buscando sustentar el perfil de una gestión fundamentada en el bienestar del trabajador como punto inicial a la concreción de los objetivos organizacionales.

En este mismo orden de ideas, Muñoz (2006) enfatiza que la instauración de un clima humanizado obliga a plantearse cuatro cuestiones fundamentales, a saber: ¿cuáles son los factores que definen a una empresa humanizada?, ¿cómo se puede detectar el grado de humanización de una empresa?, ¿qué medidas habría que llevar a cabo para cambiar hacia la humanización?, ¿cómo mantener la humanización una vez instaurada?

Por tanto, la humanización de la empresa implica no sólo la humanización del trabajo, es un concepto más amplio, que va desde el cuestionamiento de los valores culturales hasta el diseño de puestos de trabajo, pasando por cuantas cuestiones personales y sociales están involucradas en la situación laboral. En tal sentido, la empresa humanizada es aquella que ofrece al trabajador la oportunidad para el desarrollo personal y profesional.

Obliga, por tanto, a preguntarse por indicadores relacionados con la satisfacción en el trabajo, la motivación para el trabajo, el clima, la moral y la salud laboral, entre otras.

De lo planteado es posible inferir la necesidad de intervenir los espacios universitarios a través de una gestión humanizada, donde los rectores, decanos, coordinadores, personal administrativo, académico, participantes y usuarios del servicio en general, asuman el compromiso de mostrar un desempeño adecuado a las buenas prácticas, a la gestión de proyectos innovadores en materia informática, desde cuyo seno han de engendrarse aquellos proyectos, planes y programas que hagan de las universidades espacios pertinentes, de calidad, para el aprovechamiento de las potencialidades del individuo y la realización del ser. 


\section{Metodología de estudio}

Esta investigación se encuadra en un estudio de tipo documental, de análisis, lo cual de acuerdo al criterio de Finol y Camacho (2006) se define como aquel que surge de la recopilación de información a través de documentos escritos o digitales, siendo analizada de acuerdo al entender del investigador y su lógica. A la vez, se utilizó un diseño documental bibliográfico, dada la utilización de textos y referencias electrónicas.

También se utilizó la técnica de la observación documental para la recolección de datos, pudiendo hacer la revisión de los textos y documentos digitales recolectados, en el ánimo de examinarlos, analizarlos, e interpretarlos, a fin de lograr la indagación propuesta y dilucidar los contenidos dados.

Igualmente, para la interpretación de los datos recabados en este estudio se utilizó el análisis de contenido, pudiendo contrastar el significado de las temáticas seleccionadas para el investigador, de acuerdo a su experiencia, a su lógica, a su real saber y entender, con lo establecido explícita o implícitamente en los documentos revisados.

\section{Análisis desde la perspectiva de la transformación del hombre.}

En las organizaciones universitarias, según la perspectiva de Manrique (1997), el verdadero cambio se inicia con la transformación de la mente, dado que el uso permanente de modelos mentales hace a muchos comportamientos inconscientes, mientras no se tome conciencia a nivel individual de la necesidad de transformaciones sustanciales de reingeniería y reinvención. Es así como, cualquier transformación organizacional, en términos de Watzlawick, Weakland y Fisch (2009), implica necesariamente el cambio de comportamientos individuales.

Adaptando el criterio de estos autores a las universidades en estudio, podría afirmarse que, si bien en las organizaciones existen condiciones y acontecimientos que se hallan fuera de la intervención humana, sobre todo en lo atinente a la resistencia al cambio, a la carencia de insumos informáticos que simplifiquen las tareas, sí se puede considerar la manera como las personas intentan enfrentarse a estas circunstancias. Tales procesos de transformación organizacional desde la práctica tecnológica, son parte fundamental de las tareas de recursos humanos, y aún más, que estos en sí mismos se consideran una actividad innovadora de gestión humana.

Esa gestión humana tiene la oportunidad de ser protagonista en los procesos de transformación organizacional informática, ya que estos hacen posible que sea el colectivo, los miembros de la organización quienes ocupen el papel protagónico de los cambios culturales que en materia de tecnología permitirán mejorar los procesos gerenciales, administrativos y académicos, y los resultados de la organización.

Para que la universidad pueda afrontar un proceso de transformación de forma holística, se hace igualmente necesario replantear los procesos típicos llevados a cabo por la gestión humana, de modo que estos logren constituir un proceso integrado conducente al desarrollo organizacional, que le agregue valor a la misma y que 
se refleja claramente en su eficiencia. En este sentido, Nicholason, Rees y Brooks-Rooney (2010), argumentan que la gestión humana debe desempeñar un papel importante como facilitador de la innovación.

Esto puede lograrse en las universidades examinadas, a través de un proceso de sensibilización, al descentralizar la gerencia, y permitirle a los trabajadores a descubrir, emplear el conocimiento, y trabajar en equipo, de modo que permita incrementar el aprendizaje a través de interacción interdisciplinaria, dando al conocimiento su uso efectivo.

En tal sentido, debe considerarse que, para aportar a los procesos de transformación organizacional en materia de cultura tecnológica en las organizaciones, se deben implementar acciones propias de una gestión humana, debiendo ayudar a adoptar actitudes, y generar, competencias, en los cuales el pensamiento positivo e innovador, propias del talento genial, tenga lugar, pudiendo con ello renovar las metas organizacionales hacia esos fines.

\section{Reflexiones finales}

Las nuevas tendencias de gestión del talento humano en las organizaciones universitarias, desde la perspectiva de la humanización en la gestión de proyectos informáticos, muestran la capacidad de establecer estrategias que coadyuven a la excelencia de la gente en sus actividades de trabajo, mostrando un comportamiento idóneo para el aprovechamiento de potencialidades y recursos, con el compromiso, direccionado desde el esfuerzo por mantener los niveles de satisfacción en los puestos de trabajo, para dar un valor compartido a través de una gerencia con rostro humano.

Es así entonces que se requiere el desarrollo de directivos y colaboradores, como los auténticos modelos de creación de ideas en pro de la innovación y el bien común, debiendo estos ser capaces de crear un tejido social armónico, bajo un ambiente sano de corresponsabilidad de los equipos de trabajo, sustentados en una gestión en materia tecnológica como portavoz de humanización y progreso.

Lo planteado corrobora la necesidad de la implementación de las tecnologías en ambientes de modernidad, creando una sinergia entre los grupos de interés para producir el empoderamiento de la gente en sus cargos, visionados en tareas éticas, conducentes a la adquisición y la asimilación tecnológica, para reproducir los saberes y aquellos procesos tendientes a la toma de decisiones conveniente. 


\section{Bibliografía}

Alcalá, M. C. y Conti, G. (2013). La humanización del trabajo, marketing interno y la tecnología como base de la productividad social. Estudio publicado en la revista Revecitec Urbe, Volumen 4, No. 1. Disponible en http://publicaciones.urbe.edu/index.php/revecitec/ article /viewArticle/2276/4021

Calderón Hernández, G.; Cuartas Castaño, J. y Álvarez Giraldo, C. (2009). Transformación organizacional y prácticas innovadoras de gestión humana. Universidad Nacional de Colombia. Revista Innovar Journal, volumen 19, No. 35. Disponible en http://www.revistas. unal. edu.co/ index.php/innovar/article/view/28 773/ 34868.

Dolan, S. (2013). La gestión de los recursos humanos: preparando profesionales del siglo XXI. Ediciones McGraw - Hill. Madrid.

Escorsa, P.; Maspons R. y Ortiz, I. (2000). La integración entre la gestión del conocimiento y la inteligencia competitiva: la aportación de los mapas tecnológicos. Documento en línea. Disponible en: http://www.revistaespacios.com/a00v21n02/40002102.html

Finol, M. y Camacho, H. (2006). El proceso de investigación científica. Venezuela: Editorial Ediluz.

Hewitt, G. (2008). Estrategias de Empresas y RR HH. Nuevas mentalidades para Nuevos Roles. España. Gestión 2000.

Manrique, F. (1997). Un cambio de época, no una época de cambios. Bogotá: McGraw-Hill.

Morán, E. (2017). Una verdadera cultura ambiental para la transformación en las organizaciones. Desafíos actuales. Artículo no publicado, como requisito del Programa Doctorado en Ciencias Gerenciales de la Universidad Privada Dr. Rafael Belloso Chacín (URBE).

Morin, E. (1998). Para salir del Siglo XX. Barcelona: Cairos. p. $1-22$.

Muñoz, A. (2006). El futuro es la empresa humanizada. Documento en línea. Disponible en: http://coitt.es/res/revistas/antena165_08b_ articulo_futuro.pdf

Nicholason, N., Rees, A. y Brooks- Rooney, A. (2010). Estrategia, innovación y rendimiento. Revista de Estudios de Gestión, $27,511-534$.

Nuváez, J. (2016). Análisis en justicia transicional: Un razonamiento actual ineludible. Artículo publicado en la Universidad Cooperativa de Colombia (UCC), Colombia.

Pernía, J. (2017). Prospectiva Estratégica como apalancamiento de la Gestión del Capital Humano en empresas de distribución de productos de consumo masivo. Trabajo doctoral en el Programa Doctorado en Ciencias Gerenciales de la Universidad Privada Dr. Rafael Belloso Chacín (URBE). Disponible en http://www.publicacionesurbe.edu.php

Segurado, A. y Agulló, E. (2012). Calidad de vida laboral: hacia un enfoque integrador desde la psicología social. Documento en línea. Disponible en: http://www.psicothema.com/pdf/806.pdf

Universidad de Pamplona de Colombia (2016). Gestión de proyectos informáticos. Disponible en http://www.unipamplona.edu.co/ maes triagpi/

Watzlawick, P., Weakland, J.H. y Fisch, R. (2009). Cambio. Formación y solución de los problemas humanos. Barcelona: Editorial Herder. 
